\title{
Discrepancy Theory and Its Application to Finance
}

\author{
Shu Tezuka \\ IBM Tokyo Research Laboratory, \\ 1623-14, Shimotsuruma, Yamato, \\ Kanagawa, Japan 242-8502, \\ tezuka@jp.ibm.com, \\ and \\ Research Center for Advanced Economic Engineering, \\ University of Tokyo, \\ 4-6-1 Komaba, Tokyo, Japan 153-8904 \\ tezuka@aee.u-tokyo.ac.jp
}

\begin{abstract}
In this paper, we first give a brief overview of discrepancy theory, then introduce low-discrepancy sequences, in particular, the original Faure and generalized Faure sequences. Next, we describe how to apply them to the problem of pricing financial derivatives, along with a successful application of this technique to the valuation of the present value of mortgage-backed securities (MBS). Finally, we will discuss future research directions.
\end{abstract}

\section{Introduction}

Discrepancy theory is a branch of Number Theory, whose historical origin is the theory of uniform distribution developed by $\mathrm{H}$. Weyl and other mathematicians in the early days of the 20th century 126 . While the latter deals with the uniformity of infinite sequences of points, the former with the uniformity of finite sequences. Finite sequences always have some irregularity or deviation from the ideal uniformity due to their finiteness. Discrepancy is a mathematical notion for measuring such irregularity. For this reason, discrepancy theory is sometimes called as the theory of irregularities of distribution.

Quasi-Monte Carlo methods are one of the most successful applications of discrepancy theory. While Monte Carlo methods assume random numbers to provide probabilistic error bounds via the central limit theorem, quasi-Monte Carlo methods use low-discrepancy sequence 11 to allow deterministic error bounds via the Koksma-Hlawka theorem. The idea underlying low-discrepancy sequences is to use the point sets, not randomly distributed, but very uniformly distributed throughout the domain of integration. The extent to which the points are uniform has been mathematically defined as their discrepancy. The more uniformly distributed the points are, the lower the discrepancy is.

\footnotetext{
${ }^{1}$ Some people call them quasi-random sequences, but this term is a misnomer, since low-discrepancy sequences are totally deterministic.
} 
Monte Carlo simulations are a common technique in finance, particularly in derivative pricing and in $\mathrm{VaR}$ (Value at Risk) computations, since they are simple to understand and to implement. In the area of derivative pricing, stochastic differential equations have been widely used as a modeling tool to describe the time evolution of the price of the underlying financial asset. The more complex stochastic models become, the less available analytical solutions are. Thus, Monte Carlo simulations are viewed as a last resort for the computation involved in these applications. On the other hand, the more accurate the solution is required to be, the more computing time is needed. But, Monte Carlo simulations are quite unsatisfactory due to their main drawback, that is to say, their notoriously slow convergence rate. According to the central limit theorem, the convergence rate is $O(1 / \sqrt{N})$, where $N$ is the number of sample points.

The use of low-discrepancy sequences for finance problems began around 1992, by Paskov and Traub [16]. They reported that quasi-Monte Carlo methods performed very well relative to simple Monte Carlo methods, as well as to antithetic Monte Carlo methods for pricing a ten-tranche CMO (Collateralized Mortgage Obligation), which they obtained from Goldman-Sachs 15. Since then, many people (e.g., see the reference in [20]) have followed them and confirmed their finding with different pricing problems by using different types of low-discrepancy sequences.

The organization of this paper is as follows: Section 2 briefly overviews discrepancy theory: the original definition of discrepancy and its several variants. In Section 3, we introduce quasi-Monte Carlo methods, which is the deterministic version of Monte Carlo methods, and describe a general method of constructing low-discrepancy sequences, then in detail the so-called generalized Faure sequences. In Section 4, we give numerical experiments with the problem of computing the present values of MBS, along with some discussion of the results. In the last section, we conclude the paper with important research topics.

\section{Brief Overview of Discrepancy Theory}

\subsection{Definition of Discrepancy}

The formal definition of discrepancy is as follows: For $N$ points $X_{0}, X_{1}, \ldots, X_{N-1}$ in $[0,1]^{k}$, we denote

$$
\mathrm{I}(f)=\int_{[0,1]^{k}} f\left(x_{1}, \ldots, x_{k}\right) d x_{1} \cdots d x_{k}
$$

and

$$
\mathrm{Q}_{N}(f)=\frac{1}{N} \sum_{i=0}^{N-1} f\left(X_{i}\right) .
$$

We define a subinterval to be $J=\left[0, u_{1}\right) \times \cdots \times\left[0, u_{k}\right)$, where $0<u_{h} \leq 1$ for $1 \leq h \leq k$, and also define the characteristic function as $\chi_{J}(x)=1$ if 
$x \in J$; otherwise 0 . We commonly have the following two different definitions of discrepancy:

Definition 1. The $L_{\infty}$-discrepancy is defined by

$$
\left\|\mathrm{I}-\mathrm{Q}_{N}\right\|_{k, \infty} \stackrel{\text { def }}{=} \sup _{J}\left|\mathrm{I}\left(\chi_{J}\right)-\mathrm{Q}_{N}\left(\chi_{J}\right)\right|
$$

where the supremum is extended over all subintervals $J$.

Definition 2. The $L_{2}$-discrepancy is defined by

$$
\left\|\mathrm{I}-\mathrm{Q}_{N}\right\|_{k, 2} \stackrel{\text { def }}{=}\left(\int_{[0,1]^{k}}\left(\mathrm{I}\left(\chi_{J}\right)-\mathrm{Q}_{N}\left(\chi_{J}\right)\right)^{2} d u_{1} \cdots d u_{k}\right)^{1 / 2}
$$

By expanding the righthand-side, the $L_{2}$-discrepancy is explicitly written as

$$
\left\|\mathrm{I}-\mathrm{Q}_{N}\right\|_{k, 2}^{2}=\int_{I^{k}} K(x, y) d x d y-\frac{2}{N} \sum_{i=1}^{M} \int_{I^{k}} K\left(x, x_{i}\right) d x+\frac{1}{N^{2}} \sum_{i, j=1}^{M} K\left(x_{i}, x_{j}\right),
$$

where

$$
K(s, t)=\int_{I^{k}} \chi_{[0, u)}(s) \chi_{[0, u)}(t) d u=\prod_{i=1}^{k}\left(1-\max \left(s_{i}, t_{i}\right)\right) .
$$

The well known lower and upper bounds for the discrepancy are as follows: For any $N$ point set, the $L_{2}$-discrepancy satisfies

$$
\left\|\mathrm{I}-\mathrm{Q}_{N}\right\|_{k, 2}=\Omega\left(\frac{(\log N)^{(k-1) / 2}}{N}\right) .
$$

Roth (see, e.g., [16]) already proved the matching upper bound for this lower bound, although his proof was un-constructive. Regarding the $L_{\infty}$-discrepancy, we have the same lower bound as the $L_{2}$-discrepancy, but the upper bound is given as

$$
\left\|\mathrm{I}-\mathrm{Q}_{N}\right\|_{k, \infty}=O\left(\frac{(\log N)^{k-1}}{N}\right) .
$$

It is conjectured [1] that this order of magnitude in the upper bound is optimal, i.e., that the lower bound can be further improved.

\subsection{Several Variations of Discrepancy}

In what follows, we give several variations of definition of discrepancy.

Basis functions:

From the definition in equation (2), the discrepancy can be seen as an integration

${ }^{2}$ Henri Faure informed me that Chen and Skriganov recently found a constructive proof. 
error of the indicator function. So, by choosing "basis" functions different from the indicator function, we have different definitions of discrepancy. One example is due to Paskov [14, who chose

$$
f_{u}^{r}(x)= \begin{cases}(u-x)^{r} /(r !)^{k} & \text { if } u>x \\ 0 & \text { otherwise }\end{cases}
$$

as a basis function. Note that the case of $r=0$ is equivalent to the original definition (2). In this case, $K(s, t)$ in (3) is changed to

$$
K(s, t)=\int_{I^{k}} f_{u}^{r}(s) f_{u}^{r}(t) d u .
$$

If we regard the bivariate function $K(s, t)$ as a reproducing kernel on some Hilbert space, we can derive more general version of Koksma-Hlawka theorem, i.e., the worst case error estimate of integration. If we see $K(s, t)$ as a covariance kernel of some Gaussian measure, we can derive more general version of Woźniakowski theorem, the average case error estimate of integration. For more information, see Hickernell [3]. Both of Koksma-Hlawka and Woźniakowski theorems are mentioned in more detail in the next section.

\section{Subspaces:}

Another variant of the discrepancy is obtained by changing subintervals $J$ (as well as $I$ ) to be another subspace such as half space, circle(or sphere), convex etc. In terms of the order of magnitude of the discrepancy, there are roughly two families. One is that the region $J$ consists of scaled and translated copies of a fixed polygon or polytopes. The original definition is included in this family because $J$ is a class of all axis-parallel boxes, in which no rotation is allowed. For this family, the discrepancy is bounded from above and from below by some constant powers of $\log N$ divided by $N$. For the other family, we can allow rotation beside scaling and translation. For example, arbitrarily rotated rectangular boxes are included in this family. Also, this family includes a class of convex bodies, for which Schmidt obtained a lower bound as $\Omega\left(N^{-2 /(d+1)}\right)$. And the upper bound is obtained by Beck $(d=2)$ as $N^{-2 / 3} \log ^{4} N$ and by Stute $(d \geq 3)$ as $N^{-2 /(d+1)} \log ^{c} N$, where $c=1.5$ for $d=3$ and $c=2 /(d+1)$ for $d \geq 4$. For this family, the discrepancy is bounded by the order of some negative fractional power of $N$. See 16 for more details.

\section{Nonuniform weights:}

Another interesting generalization of the definition of discrepancy is the use of non-uniform (or unequal) weights. Then, the equation (1) is replaced by

$$
\mathrm{Q}_{N, w}(f)=\sum_{i=0}^{N-1} w_{i} f\left(X_{i}\right)
$$

where $w_{i}$ are all real numbers. If $w_{i}=1 / N$ for all $i$, the original definition follows. This kind of definition is particularly useful for the error analysis in numerical 
integration, where non-uniform weights are commonly used in quadrature (and cubature) formulas, e.g., Simpson rule, Gauss rule, etc. By exploiting this definition, Wasilkowski and Woźniakowski 22] obtained the following remarkable result:

Theorem 1. There exists a cubature formula $Q_{N, w}$ such that

$$
\left\|\mathrm{I}\left(\chi_{J}\right)-\mathrm{Q}_{N, w}\left(\chi_{J}\right)\right\|_{k, 2} \leq \epsilon
$$

for $N(\epsilon, k)$ satisfying

$$
N(\epsilon, k) \leq A \epsilon^{-1.4778 .}
$$

where $A$ is an absolute constant.

If we compare the above result with the Monte Carlo methods, which need the number of sample points as

$$
N(\epsilon, k)=O\left(\epsilon^{-2}\right)
$$

to achieve the standard deviation of $\epsilon$, then its significance becomes clear. Another important point is that $A$ is not depending on the dimension $k$. No similar results have been so far obtained for the discrapncy with uniform weights.

\section{Combinatorial discrepancy:}

The most abstract variant of the definition of the discrepancy is combinatorial discrepancy (or red-blue discrepancy): Let $X$ be a set with cardinality $n$, and let $S$ be a system of subsets of $X$. A mapping $\chi: X \rightarrow\{-1,1\}$ is called a coloring. The discrepancy of $S$ is defined as

$$
\operatorname{disc}(S)=\min _{\chi} \operatorname{disc}(S, \chi)
$$

where $\operatorname{disc}(S, \chi)=\max _{A \in S}|\chi(A)|$ and $\chi(A)=\sum_{x \in A} \chi(x)$. The following upper bound can be derived by using a random coloring:

Theorem 2. We have

$$
\operatorname{disc}(S)=O(\sqrt{n \log m}),
$$

where we assume $A$ have at most $m$ subsets.

Below, we give two typical examples of a set system $(X, S)$.

Example 1. Consider a graph $(V, E)$. Let $V$ be a set $X$, and $S$ be a set of subsets $A(v)$, where $A(v)$ denotes a set of neighboring nodes of $v \in V$. Then, the discrepancy with respect to this set system $(V, S)$ is known as a very useful measure for the graph coloring problem, which play an important role in theoretical computer science.

Example 2. Consider the set $X=\{1,2, \ldots, n\}$ and let $S$ be a set of all subsets $\{a, a+b, a+2 b, \ldots\} \cap X$, where $a$ and $b$ are any integers. The discrepancy with respect to this set system $(X, S)$ is known closely related to a famous Van der Waerden theorem in number theory.

More details and further discussions on discrepancy in general, see Matousek's excellent book[6]. 


\section{Quasi-Monte Carlo Methods}

\subsection{Numerical Integration and Low-Discrepancy Sequences}

Finance-related Monte Carlo problems, particularly those related to derivative pricing, can be formulated as problems of computing multidimensional integrals. The dimension of the integration is usually equal to the number of time steps by which the time interval under consideration is discretized. Once a problem can be formulated as one of numerical multidimensional integration, we have several "deterministic" cubature formulas. However, the direct extensions of one-dimensional quadrature formulas, such as trapezoidal rules, to higherdimensional ones do not work well, because of the curse of dimensionality [21]. For example, the error bound for the $k$-dimensional trapezoidal rules is known to be $O\left(N^{-2 / k}\right)$, which means that the error grows exponentially as the dimension size becomes larger.

Low-discrepancy sequences have been considered as a promising alternative to high-dimensional numerical integration because its asymptotic error bound is $O\left((\log N)^{k} / N\right)$ for the $k$-dimensional integration problem. The Koksma-Hlawka theorem gives an important relation between discrepancy and numerical integration [8]:

Theorem 3 (Koksma-Hlawka). If the integrand $f$ is of bounded variation on the $k$-dimensional unit hypercube $[0,1]^{k}$ in the sense of Hardy and Krause, then for any $X_{0}, X_{1}, \ldots, X_{N-1} \in[0,1)^{k}$ we have

$$
\left|\mathrm{I}(f)-\mathrm{Q}_{N}(f)\right| \leq\left\|\mathrm{I}-\mathrm{Q}_{N}\right\|_{k, \infty}\|f\|,
$$

where $\|f\|$ is the Hardy-Krause variation of $f$.

What is most important here is that the bound is a product of two separate elements: one is dependent only on the point set and independent on $f$; the other only on the integrand and independent on the point set. For a given integrand $f$, if we can choose a point set so as to make the discrepancy as small as possible, we have the smallest integration error. We also have another important result, the Woźniakowski theorem [23]:

Theorem 4 (Woźniakowski). Let $C_{k}$ be the class of real continuous functions defined on $[0,1]^{k}$ equipped with the classical Wiener sheet measure $w$ (that is, Gaussian with mean zero and covariance kernel

$$
R(\mathbf{s}, \mathbf{t}) \stackrel{\text { def }}{=} \int_{C_{k}} f(\mathbf{s}) f(\mathbf{t}) w(d f)=\min (\mathbf{s}, \mathbf{t}) \stackrel{\text { def }}{=} \prod_{j=1}^{k} \min \left(s_{j}, t_{j}\right)
$$

for any vectors $\mathbf{s}=\left(s_{1}, \ldots, s_{k}\right)$ and $\mathbf{t}=\left(t_{1}, \ldots, t_{k}\right)$ in $\left.[0,1]^{k}\right)$. Then, for a given set of points $X_{i}=\left(x_{i 1}, \ldots, x_{i k}\right), i=0,1, \ldots, N-1$, in $[0,1]^{k}$, we have

$$
\int_{C_{k}}\left(\mathrm{I}(f)-\mathrm{Q}_{N}(f)\right)^{2} w(d f)=\left\|\mathrm{I}-\mathrm{Q}_{N}\right\|_{k, 2, J}^{2},
$$

where $\left\|\mathrm{I}-\mathrm{Q}_{N}\right\|_{k, 2, J}$ is defined as the $L_{2}$-discrepancy with $J=\left(u_{1}, 1\right] \times \cdots \times$ $\left(u_{k}, 1\right]$ in equation (2), where $0 \leq u_{h}<1$ for $1 \leq h \leq k$. 
The theorem means that on average the integration error is dependent only on the discrepancy, not on the integrand $f$. Both of the above theorems tell us that the lower the discrepancy is, the smaller the integration error will be.

We now introduce the formal definition of low-discrepancy sequences:

Definition 3. If a sequence $X_{0}, X_{1}, \ldots$ in $[0,1]^{k}$ satisfies the condition that for all $N>1$, the discrepancy of the first $N$ points is

$$
\left\|\mathrm{I}-\mathrm{Q}_{N}\right\|_{k, \infty} \leq C_{k} \frac{(\log N)^{k}}{N}
$$

where $C_{k}$ is a constant depending only on the dimension $k$, then we call it a low-discrepancy sequence.

Notice that the order of magnitude $(\log N)^{k} / N$ in the right-hand side is believed to be the optimal upper bound. Therefore, the sole difference among the many types of low-discrepancy sequences is how small the constant factor $C_{k}$ is. In this article, we concentrate on the construction of low-discrepancy sequences based on $(t, k)$-sequences in base $b$. Before introducing them, we need the following definitions:

Definition 4. A $b$-ary box is an interval of the form

$$
E=\prod_{h=1}^{k}\left[a_{h} b^{-d_{h}},\left(a_{h}+1\right) b^{-d_{h}}\right)
$$

with integers $d_{h} \geq 0$ and integers $0 \leq a_{h}<b^{d_{h}}$ for $1 \leq h \leq k$.

Definition 5. Let $0 \leq t \leq m$ be an integer. A $(t, m, k)$-net in base $b$ is a point set of $b^{m}$ points in $[0,1]^{k}$ such that every $b$-ary box of volume $b^{t-m}$ contains exactly $b^{t}$ points of the point set.

Now, we define $(t, k)$-sequences in base $b$.

Definition 6. Let $0 \leq t \leq m$ be an integer. A sequence of points $X_{0}, X_{1}, \ldots$, in $[0,1]^{k}$ is called a $(t, k)$-sequence if for all integers $j \geq 0$ and $m>t$, the point set consisting of $\left[X_{n}\right]_{m}$ with $j b^{m} \leq n<(j+1) b^{m}$ is a $(t, m, k)$-net in base $b$, where $[X]_{m}$ denotes the coordinate-wise $m$-digit truncation in base $b$ of $X$.

Following Sobol' and Faure's results, Niederreiter [8] obtained the following theorem for an arbitrary integer base $b \geq 2$ :

Theorem 5 (Niederreiter). For any $N>1$, the discrepancy of the first $N$ points of a $(t, k)$-sequence in base $b$ satisfies

$$
\left\|\mathrm{I}-\mathrm{Q}_{N}\right\|_{k, \infty} \leq c(t, k, b) \frac{(\log N)^{k}}{N}+O\left(\frac{(\log N)^{k-1}}{N}\right),
$$

where $c(t, k, b) \approx \frac{b^{t}}{k !}\left(\frac{b}{2 \log b}\right)^{k}$ 
This means that if $t$ and $b$ are constant or depend only on $k$, then the $(t, k)$ sequence becomes a low-discrepancy sequence. Note that a smaller value of $t$ gives a lower discrepancy asymptotically. Thus, a $(0, k)$-sequence can be said as the best in this sense. We should notice that any subset of $s<k$ coordinates of $(0, k)$-sequences constitutes $(0, s)$-sequences.

\subsection{Generalized Faure Sequences}

Niederreiter presented a general construction principle for $(t, k)$-sequences as follows: Let $k \geq 1$ and $b \geq 2$ and $B=\{0,1, \ldots, b-1\}$. Accordingly, we define

(i) a commutative ring $R$ with identity and $\operatorname{card}(R)=b$;

(ii) bijections $\psi_{j}: B \rightarrow R$ for $j=1,2, \ldots$, with $\psi_{j}(0)=0$ for all sufficiently large $j$;

(iii) bijections $\lambda_{h i}: R \rightarrow B$ for $h=1,2, \ldots, k$ and $i=1,2, \ldots$, with $\lambda_{h i}(0)=0$ for $1 \leq h \leq k$ and all sufficiently large $i$;

(iv) elements $c_{i j}^{(h)} \in R$ for $1 \leq h \leq k, 1 \leq i, 1 \leq j$, where for fixed $h$ and $j$ we have $c_{i j}^{(h)}=0$ for all sufficiently large $i$.

For $n=0,1,2, \ldots$, write $n=\sum_{r=1}^{\infty} a_{r}(n) b^{r-1}$ with $a_{r}(n) \in B$. For $h=1, \ldots, k$, set the $h$-th coordinate of the point $X_{n}$ in $[0,1]^{k}$ as

$$
X_{n}^{(h)}=\sum_{i=1}^{\infty} x_{n i}^{(h)} b^{-i}
$$

where

$$
x_{n i}^{(h)}=\lambda_{h i}\left(\sum_{j=1}^{\infty} c_{i j}^{(h)} \psi_{j}\left(a_{j}(n)\right)\right) \in B
$$

for $1 \leq h \leq k, 1 \leq i$, and $0 \leq n$. We call $C^{(h)}=\left(c_{i j}^{(h)}\right)$ the generator matrix for the $h$-th coordinate of a $(t, k)$-sequence.

We now describe how to construct such generator matrices so that we obtain low-discrepancy sequences called generalized Niederreiter sequences (see Tezuka [19] 3. The construction is based on $G F\{b, z\}$, i.e., the formal Laurent series expansions over the finite field $G F(b)$, where $b$ is a prime power. Denote $S(z) \in$ $G F\{b, z\}$ by

$$
S(z)=\sum_{j=w}^{\infty} a_{j} z^{-j},
$$

where all $a_{j} \in G F(b)$ and $w$ is an arbitrary integer. Hereafter, we use the following notations: $[S(z)]$ denotes the polynomial part of $S(z)$ and $[S(z)]_{p(z)} \stackrel{\text { def }}{=}$ $[S(z)] \quad(\bmod p(z))$ with $0 \leq \operatorname{deg}\left([S(z)]_{p(z)}\right)<\operatorname{deg}(p(z))$.

${ }^{3}$ In 1995, Niederreiter and Xing [9] presented a new construction method of lowdiscrepancy sequences based on algebraic function fields. Today, their sequences are known as the "theoretically" best, but there are several implementation issues to be overcome before these sequences become available for practical use. 
Let $k$ polynomials $p_{1}(z), \ldots, p_{k}(z)$ be pairwise coprime and let $e_{h}=\operatorname{deg}\left(p_{h}\right) \geq$ 1 for $1 \leq h \leq k$. For $m \geq 1,1 \leq h \leq k$, and $j \geq 1$, consider the expansion

$$
\frac{y_{h m}(z)}{p_{h}(z)^{j}}=\sum_{r=w}^{\infty} a^{(h)}(j, m, r) z^{-r}
$$

by which the elements $a^{(h)}(j, m, r) \in G F(b)$ are determined. Here $w \leq 0$ may depend on $h, j, m$, and each $y_{h m}(z)$ is a polynomial such that the residue polynomials $\left[y_{h m}(z)\right]_{p_{h}(z)},(j-1) e_{h} \leq m-1<j e_{h}$, are linearly independent over $G F(b)$ for any $j>0$ and $1 \leq h \leq k$. Then define

$$
c_{m r}^{(h)}=a^{(h)}\left(m_{h}+1, m, r\right)
$$

for $1 \leq h \leq k, m \geq 1$, and $r \geq 1$, where $m_{h}=\left[(m-1) / e_{h}\right]$.

Tezuka [19] proved the following theorem:

Theorem 6. If an integer $t \geq 0$ satisfies $t \geq \sum_{h=1}^{k} \operatorname{deg}\left(p_{h}\right)-k$, then the generalized Niederreiter sequence becomes a $(t, k)$-sequence in base $b$.

Remark 1. Faure sequences are $(0, k)$-sequences in a prime base $b \geq k$ obtained from generalized Niederreiter sequences such that all $y_{h m}(z)=1$.

This remark motivates the following definition [1819]:

Definition 7. Generalized Faure sequences are defined as $(0, k)$-sequences obtained from generalized Niederreiter sequences.

In practice, we choose the base $b$ to be prime, for which we have the following matrix representation for all generator matrices $C^{(h)}, 1 \leq h \leq k$ :

$$
C^{(h)}=A^{(h)} P^{h-1},
$$

where $A^{(h)}, 1 \leq h \leq k$, are nonsingular lower triangular matrices over $G F(b)$ and $P$ is the Pascal matrix whose $(i, j)$ element is equal to $\left(\begin{array}{l}j-1 \\ i-1\end{array}\right)$. The original Faure sequences correspond to the case in which $A^{(h)}=I$ for all $h$.

\section{Applications to Finance}

In this section, we apply quasi-Monte Carlo methods to a problem related to pricing Mortgage Backed Securities (MBS) originally described by Paskov [15]. In the experiment, we used the original Faure sequence and a generalized Faure sequence with base $b=367$. More precisely, nonsingular lower triangular matrices $A^{(h)}, 1 \leq h \leq k$, in equation (4) for the generator matrices were chosen at random, and we omitted the first 100000 points; that is to say, we used the points $X_{i}, i=100001,100002, \ldots$, of the sequence. 


\section{Mortgage-Backed Securities}

MBS, the most popular fixed income derivatives, are a kind of interest-rate option, whose underlying asset is a pool of residential mortgage portfolios 4]. They have a critical feature of prepayment privileges, because householders can prepay their mortgages at any time. The integration problem associated with MBS is summarized as follows: We use the following notation:

$r_{k}$ : the appropriate interest rate in month $k$

$w_{k}$ : the percentage prepaid in month $k$

$a_{k}$ : the remaining annuity after $361-k$ months

$C$ : the monthly payment on the underlying mortgage pool

for $k=1,2, \ldots, 360$, where $a_{k}=1+d_{1}+\cdots+d_{1}^{k-1}$ is constant with $d_{1}=1 /\left(1+r_{0}\right)$ and $r_{0}$ is the current monthly interest rate. $C$ is also constant. The variable $r_{k}$ follows the discrete-time version of the following geometric Brownian motion:

$$
\log r_{k}-\log r_{k-1}=\left(a-\frac{\sigma^{2}}{2}\right) \Delta+\sigma d B,
$$

where $\Delta=1$ and $d B$ is the normal random variable with mean zero and variance $\Delta$. Here, we assume zero drift (i.e., $a=0$ ) in order to make $E\left(r_{k}\right)=r_{0}$ for $k=1, \ldots, 360$. Thus,

$$
r_{k}=K_{0} \exp \left(\sigma z_{k}\right) r_{k-1}, \text { for } k=1,2, \ldots, 360,
$$

where $z_{k}, k=1,2, \ldots, 360$, are independent standard normally distributed random variables, and $K_{0}=\exp \left(-\sigma^{2} / 2\right)$.

The prepayment model for the variables $w_{k}, k=1,2, \ldots, 360$, depends on the interest rate $r_{k}, k=1,2, \ldots, 360$, as follows:

$$
w_{k}=K_{1}+K_{2} \arctan \left(K_{3} r_{k}+K_{4}\right),
$$

where $K_{1}, K_{2}, K_{3}$, and $K_{4}$ are given constants. In practice, the lower the interest rate is, the higher the prepayment rate becomes. Thus, the cash flow in month $k$ is

$$
M_{k}\left(z_{1}, \ldots, z_{k}\right)=C\left(1-w_{1}\right) \cdots\left(1-w_{k-1}\right)\left(1-w_{k}+w_{k} a_{361-k}\right) .
$$

This is multiplied by the discount factor

$$
d_{k}\left(z_{1}, \ldots, z_{k-1}\right)=\prod_{i=0}^{k-1} \frac{1}{1+r_{i}} .
$$

We have the following total present value of MBS:

$$
P V\left(z_{1}, \ldots, z_{360}\right)=\sum_{k=1}^{360} d_{k}\left(z_{1}, \ldots, z_{k-1}\right) M_{k}\left(z_{1}, \ldots, z_{k}\right) .
$$

What we want to compute is the expected value of the present value $P V$ over all independent random variables $z_{k}, k=1, \ldots, 360$. By using the inversion of 
the normal distribution, we can formulate this problem as one of computing a multivariate integration over $[0,1]^{360}$ :

$$
\mathrm{E}(P V)=\int_{[0,1]^{360}} P V\left(u_{1}, \ldots, u_{360}\right) d u_{1} \cdots d u_{360},
$$

where $u_{k}=\mathrm{N}\left(z_{k}\right)$ for $k=1, \ldots, 360$.

In this experiment, we used the parameter set $\left(r_{0}, K_{1}, K_{2}, K_{3}, K_{4}, \sigma\right)=$ $(.00625, .24, .134,-261.17,12.72, .2)$ from [20], where the expected value of $P V$ is numerically computed as $143.0182 \times C$ by using more than one million sample paths. Figure 1 shows the convergence of Monte Carlo and quasi-Monte Carlo. The solid line (MBS.MC) shows the result obtained by the Monte Carlo method, while the two different dotted lines (MBS.faure and MBS.gfaure) show the results obtained by the two quasi-Monte Carlo methods. Here, we stress two important points: (1) Monte Carlo vs. quasi-Monte Carlo (generalized Faure), and (2) original Faure vs. generalized Faure. For comparison, if we look at 1000 samples, generalized Faure sequences converge to the correct value within an accuracy of $10^{-5}$, that is, $143.0182 \pm 0.00143$. On the other hand, the standard deviation of $P V$ computed from the first 1000 sample values of the Monte Carlo simulation is about 0.276 . Thus, the $99 \%$ confidence interval is about $143.0182 \pm 2.575 * 0.276 / \sqrt{1000}=143.0182 \pm 0.0225$. Therefore, we can say that about 250 times speed-up was gained by quasi-Monte Carlo with the generalized Faure sequence for this problem. On the other hand, from the comparison between Monte Carlo and quasi-Monte Carlo with the original Faure, we see the performance of the convergence looks similar.

\section{Conclusion}

Concluding the paper, I would like to present the following two interesting questions:

\section{$M C$ vs. $Q M C$}

Why does QMC outperform MC so significantly for high-dimensional numerical integration associated with finance problems? One idea to explain this result is the "effective dimension" [15,17 21], which comes from the observation that in many finance problems a small number of variables (say, near-future interest rates) are very crucial for the payoff function, while all the others are much less important. Thus, it is thought that even if the nominal dimension is large, say 360 for finance problems, the effective dimension should be small. This sounds why QMC was so successful for finance problems with large dimensions. Several proposals for the formal and quantitative definitions of the effective dimension have been made by several authors, but, at this moment, no decisive one is yet available to clearly explain the QMC's excellent performance in finance.

Papageorgiou [1113] recently found that QMC also works very well compared with MC for isotropic problems in Physics. And Tezuka [20] pointed out 


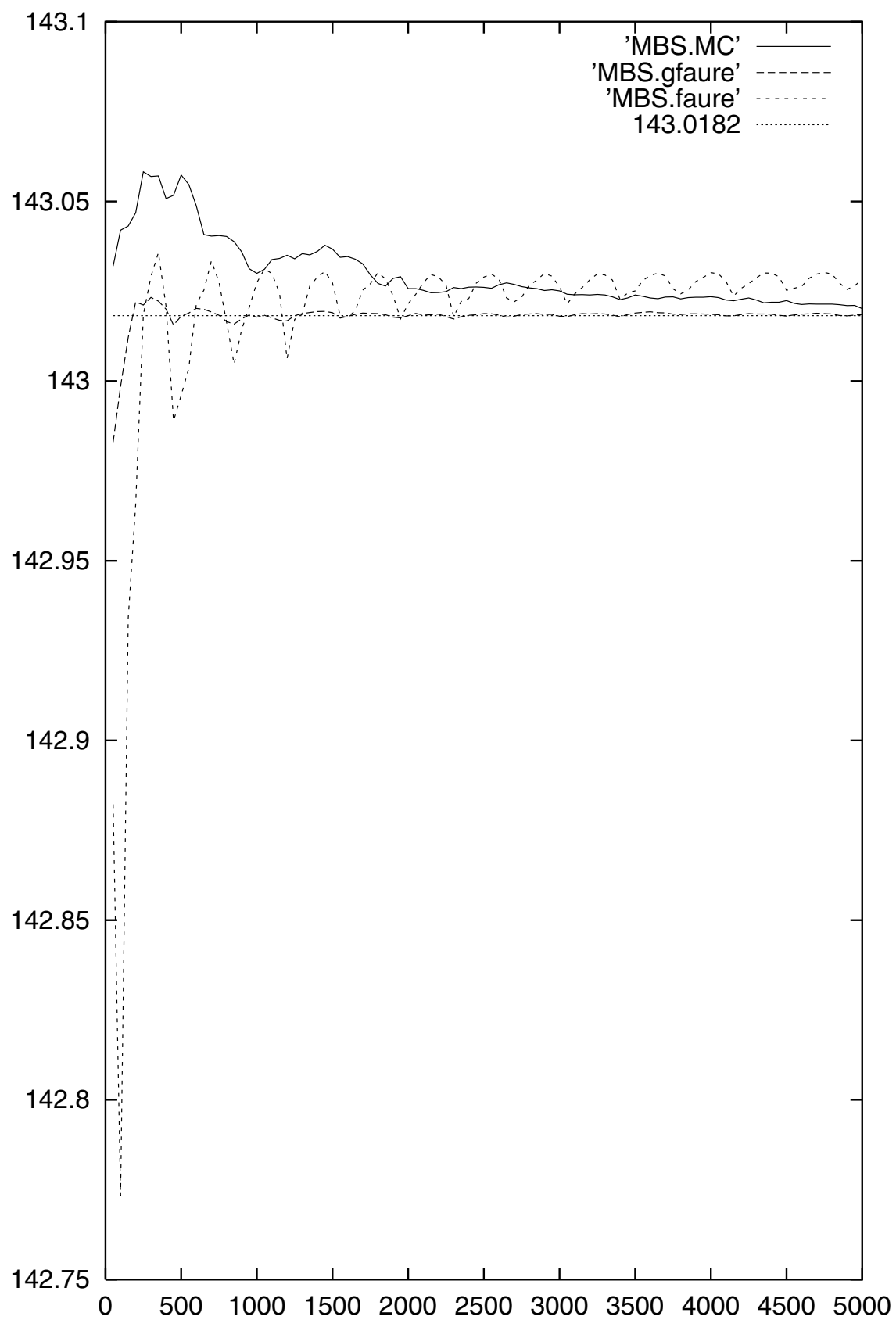

Fig. 1. Convergence of Monte Carlo and quasi-Monte Carlo methods for MBS (price vs. sample paths) 
that there also exists an isotropic problem in finance for which QMC outperforms MC. In these cases, the idea of effective dimension does not work because the importance of all the variables is the same for the isotropic integrand. The problem of how to explain these results is another important research topic.

\section{Faure vs. GFaure}

Among many classes of low-discrepancy sequences, the practical behaviors of convergence rate are different for one from another, even if the theoretical convergence rates, i.e., the upper bounds of the discrepancy, are exactly the same. A striking example of this phenomenon is the difference between practical convergence behaviors of the original Faure and generalized Faure sequences as shown in the preceding section.

For generalized Faure sequences, the effect of the use of lower triangular matrices $A^{(h)}$ on the convergence rate has been theoretically and empirically studied by several researchers $5[610,12$. Assuming that the integrand is a fixed sufficiently smooth real function, their results imply that the expected integration error over all generalized Faure sequences becomes asymptotically

$$
O\left(\frac{(\log N)^{(k-1) / 2}}{N^{3 / 2}}\right) \text {. }
$$

We should notice that the denominator $N^{3 / 2}$ is much larger than $N$, which is well-known for ordinary QMC. Unfortunately, this is an asymptotic result. We need more elaborate on this for practical size of $N$ and $k$. From another viewpoint, this can be interpreted as an existence theorem of a very good generalized Faure sequence for numerical multidimensional integration. Derandomization for finding such good sequences is very interesting in both theory and practice of low-discrepancy sequences.

\section{Acknowledgments}

I thank Professor Takayasu Ito for inviting me to the IFIP-TCS Conference in Sendai on August 17-19, 2000.

\section{References}

1. J. Beck and W. L. Chen, Irregularities of Distribution, Cambridge University Press, Cambridge, 1987.

2. M. Drmota and R. F. Tichy, Sequences, Discrepancies and Applications, Lecture Notes in Mathematics 1651, Springer-Verlag, Berlin, 1997.

3. F. J. Hickernell, Lattice Rules: How Well Do They Measure Up, in Random and Quasi-Random Point Sets, editted by P. Hellekalek and G. Larcher, Lecture Notes in Statistics, 138, Springer, New York (1998), 109-166.

4. J. C. Hull, Options, Futures, and Other Derivatives, 3rd Edition, Prentice-Hall, New Jersey, 1997. 
5. J. Matoušek, On the $L_{2}$-Discrepancy for Anchored Boxes, Journal of Complexity, 14 (1998), 527-556.

6. J. Matoušek, Geometric Discrepancy: An Illustrated Guide, Springer,1999.

7. H. Niederreiter, Quasi-Monte Carlo Methods and Pseudorandom Numbers, Bull. Amer. Math. Soc., 84 (1978), 957-1041.

8. H. Niederreiter, Random Number Generation and Quasi-Monte Carlo Methods, CBMS-NSF Regional Conference Series in Applied Mathematics, No. 63, SIAM, 1992.

9. H. Niederreiter and C. Xing, Low-Discrepancy Sequences and Global Function Fields with Many Rational Places, Finite Fields and Their Applications, 2 (1996), 241-273.

10. A. Owen, Monte-Carlo Variance of Scrambled Net Quadrature, SIAM J. Numer. Analysis, 34 (1997), 1884-1910.

11. A. Papageorgiou, Fast Convergence of Quasi-Monte Carlo for a Class of Isotropic Integrals, to appear in Math. Comp..

12. A. Papageorgiou and J. F. Traub, Beating Monte Carlo, RISK, 9 (June 1996), 63-65.

13. A. Papageorgiou and J. F. Traub, Faster Evaluation of Multidimensional Integrals, Computers in Physics, 11 (Nov/Dec 1997), 574-578.

14. S. H. Paskov, Average Case Complexity of Multivariate Integration for Smooth Functions, Journal of Complexity, 9 (1993), 291-312.

15. S. H. Paskov, New Methodologies for Valuing Derivatives, in Mathematics of Derivative Securities, edited by M. A. H. Dempster and S. Pliska, Isaac Newton Institute, Cambridge University Press, Cambridge UK (1997), 545-582.

16. S. H. Paskov and J. F. Traub, Faster Valuation of Financial Derivatives, Journal of Portfolio Management, 22:1 (Fall 1995), 113-120.

17. I. H. Sloan and H. Woźniakowski, When are Quasi-Monte Carlo Algorithms Efficient for High Dimensional Integrals, Journal of Complexity 14 (1998), 1-33.

18. S. Tezuka, A Generalization of Faure Sequences and its Efficient Implementation, Research Report RT-0105,IBM Tokyo Research Laboratory, 1994.

19. S. Tezuka, Uniform Random Numbers: Theory and Practice, Kluwer Academic Publishers, Boston, 1995.

20. S. Tezuka, Financial Applications of Monte Carlo and Quasi-Monte Carlo Methods, in Random and Quasi-Random Point Sets, editted by P. Hellekalek and G. Larcher, Lecture Notes in Statistics, 138, Springer, New York (1998), 303-332.

21. J. F. Traub and A. G. Werschulz, Complexity and Information, Cambridge Univ. Press, 1998.

22. G. Wasilkowski and H. Woźniakowski, The Exponent of Discrepancy is at Most 1.4778..., Math. Comp., 66 (1997), 1125-1132.

23. H. Woźniakowski, Average Case Complexity of Multivariate Integration, Bull. Amer. Math. Soc. , 24 (1991), 185-194. 